September 2014, Volume 2

Proceeding of $3^{\text {rd }}$ International Conference on Computation for Science and Technology, ISSN xxxx-yyyy

\title{
Binding Models of Polyphenols to Cytochrome P450 2C9: A Molecular Docking Study
}

\author{
Siripat Chaichit, Darunee Hongwiset, Supat Jiranusornkul ${ }^{*}$ \\ Laboratory for Molecular Design and Simulation (LMDS), Department of Pharmaceutical Sciences, Faculty of Pharmacy, Chiang Mai \\ University, Chiang Mai, Thailand
}

Received: 6 October 2014 / Accepted: 30 November 2014

\begin{abstract}
:
Polyphenols are widely presented in plants and dietary supplements. The beneficial effects of these compounds have been demonstrated, including the prevention of cardiovascular diseases, osteoporosis, neurogenerative diseases and diabetes. From previous studies, polyphenols showed the inhibitory effect on cytochrome P450 2C9 (CYP2C9) resulting in clinically significant of herb-drug interactions. CYP2C9 is involved in the biotransformation of various therapeutic drugs, including tolbutamide, phenytoin and warfarin. Co-administration of polyphenols with drugs that are metabolized through CYP2C9 can cause therapeutic failures or adverse events from the co-administered drugs. This study aimed to identify the interactions between polyphenols and CYP2C9 by using molecular docking program, CDOCKER, to calculate binding energy. Ten polyphenols used in this study were naringenin, chrysin, apigenin, luteolin, acacetin, galangin, kaempferol, quercetin, myricetin and isorhamnetin. The results showed that polyphenols can bind to the same binding site as flurbiprofen, which was used as the ligand in the x-ray crystal structure of the CYP2C9-flurbiprofen complex (1R9O) and the amino acid Phe100, Pro101, Glu104, Phe114, Leu208, Gly296, Ala297, Leu366 and Phe476 residues play a key role in the CYP2C9 active binding site.
\end{abstract}

Key words: CDOCKER, CYP2C9, docking, flurbiprofen, interaction, polyphenols

\section{Introduction}

Polyphenols are the second metabolites compounds which are widely distributed in plants. They are presented in vegetables, fruits, beverages and dietary supplements [1]. Polyphenols can be classified into many groups depend on the basis structure such as phenolic acids, flavonoids, stilbenes and lignans [2].The epidemiologic studies of dietary polyphenols suggest that the consumption of the polyphenol-rich foods have shown the beneficial effects to human health such as the prevention of cardiovascular diseases, osteoporosis, neurogenerative diseases and diabetes [3].

Cytochrome P450 enzymes (CYPs) are isozymes in human liver microsomes, which play important roles in the oxidative metabolism ofendogenous compounds and clinical drugs [4]. The most important CYP450 isoforms including CYP1A2, 2C9, 2C19, 2D6, 2E1 and 3A4 are responsible for the metabolism of $>90 \%$ clinical drugs [5]. Among CYP450 isoforms, CYP2C9is one of the important major-drug metabolizing isoform which metabolizes $20 \%$ of clinical drugs such as (S)-warfarin, tolbutamide, phenytoin and non-steroid antiinflammatory drugs [6].

Literatures have shown the inhibition of CYP2C9 by polyphenols may cause clinically important herb-drug interactions. Hence, this work aims to study the binding models of polyphenol to $\mathrm{CYP} 2 \mathrm{C} 9$ by a molecular docking method and identify the key residues in polyphenol-CYP2C9 interaction.

\section{Experimental}

\section{Ligands and receptor preparing}

The 3D structures of ten polyphenols such asnaringenin, chrysin, apigenin, luteolin, galangin, kaempferol, quercetin, myricetin, isorhamnetin and acacetin (Figure 1) were constructed applying ChemBioOffice and minimized using the MM2 force field and protonated at physiological $\mathrm{pH}$ by Discovery Studio 2.5.

The crystal structure of cytochrome P450 2C9 cocystallized with flurbiprofen (PDB ID: 1R9O) [7] was obtained from RCSB Protein Data Bank. The protein was prepared for docking by removing water molecules, flurbiprofen (FLP) and glycerol (GOL).

\section{Docking study}

In this study, molecular docking was performed to define the binding models of the interactions between polyphenolic compounds and CYP2C9.

The CHARMm-based Docker (CDOCKER) [8] program from Discovery Studio 2.5 was used to estimate binding energy andinvestigate the orientations of polyphenols within CYP2C9 active site.

\footnotetext{
*Corresponding author: Supat Jiranusornkul,

E-mail: supat.jira@cmu.ac.th
} 
<smiles>Nc1cc(O)cc2c1C(=O)C[C@@H](c1ccc(O)cc1)O2</smiles><smiles>O=c1cc(-c2ccccc2)oc2cc(O)cc(O)c12</smiles>
Chrysin<smiles>O=c1cc(-c2ccc(O)cc2)oc2cc(O)cc(O)c12</smiles>

Apigenin<smiles>O=c1cc(-c2ccc(O)c(O)c2)oc2cc(O)cc(O)c12</smiles>

Luteolin<smiles>COc1ccc(-c2cc(=O)c3c(O)cc(O)cc3o2)cc1</smiles>

Acacetin<smiles>O=c1c(O)c(-c2ccc(O)c(O)c2)oc2cc(O)cc(O)c12</smiles><smiles>O=c1c(O)c(-c2ccccc2)oc2cc(O)cc(O)c12</smiles>

Galangin<smiles>N#[Y16]c1cc(O)cc2oc(-c3cc(O)c(O)c(O)c3)c(O)c(=O)c12</smiles><smiles>[Y]#CC(=O)c1c(O)cc(O)cc1Oc1ccc(O)cc1</smiles>

Kaempferol

Figure 1. The structure of polyphenols.

All prepared ligands were docked into the active site, which was created as a spherical region with a diameter of $26 \AA$. The docking parameters were set with 50 top hits, 100 random conformations and 1000 random conformation dynamics steps. Final docking conformations were clustered, based on a heavy atoms with the RMSD less than $1.0 \AA$.

\section{Results and Discussion}

To determine the reliability of docking system, the docking protocol by CDOCKER was evaluated. Flurbiprofen, which was the co-crystallized ligand of CYP2C9 (1R9O), was initially re-docked into the active site. Ten prepared polyphenols were then docked into the active site of CYP2C9.

The docking result was shown in Table 1 . The mean binding energy $(\mathrm{kcal} / \mathrm{mol})$ of the polyphenols, namely, naringenin, chrysin, apigenin, luteolin, acacetin, galangin, kaempferol, quercetin, myricetin and isorhamnetin, are $-22.70,-20.26,-25.27,-31.16,-21.76,-13.41,-16.88$, $-21.41,-22.04$ and $-13.62 \mathrm{kcal} / \mathrm{mol}$, respectively. The lowest binding energy from 100 docked structures of each polyphenol are also presented in the table 1 . Kimura and co-workers have indicated that polyphenols exhibited the inhibitory activities to CYP2C9 [9]. Most flavones and flavonols showed strong inhibitory activities (>80\% inhibition) on CYP2C9. Furthermore, the polyphenols including kaempferol, isorhamnetin, galangin and naringenin, revealed a potent inhibitory effect (>100\% inhibition) against CYP2C9 with IC50 values at $12.6,15.8,7.5$, and $12.4 \mu \mathrm{M}$, respectively. Moreover, $\mathrm{Si}$ and co-workers showed that luteolin, apigenin and chrysin were potent inhibitors of CYP2C9, with $\mathrm{Ki} \leqslant 2.2 \mu \mathrm{M}[10]$.

The orientation of ten polyphenols to CYP2C9 suggests that they are the same binding site as flurbiprofen site. The biphenyl of polyphenols is closed to the haem at less than $7 \AA$. The major favored binding conformations of polyphenols in active site of CYP2C9 are shown in Figure 2.

From the CYP2C9-polyphenol complexes, the amino acid Phe100, Pro101, Glu104, Phe114, Leu208, Gly296, Ala297, Leu366 and Phe476 residues play a key role in the CYP2C9 active binding site.

According to theresults (Table 1.), flavones (luteolin and apigenin) have shown the lower binding energy than flavanones (naringenin) and flavonols (galangin, kaempferol, isorhamnetin). Based on the basis structure of flavones, they have double bond between $\mathrm{C} 2$ and $\mathrm{C} 3$ [11]. As the flavones luteolin with the lowest binding energy $(-31.16 \mathrm{kcal} / \mathrm{mol})$, has the hydroxyl group at 3'and 4'- position of B-ring and apigenin has a hydroxyl group at 4'-position of B-ring whereas chrysinand acacetin, with no hydroxyl groups at B-ring, have weak binding energy among the flavones. The docked orientation of luteolin in CYP2C9 indicates that the oxygen atom on B-ring is able to form hydrogen bond between Leu102 and Glu104. Therefore, this suggests that the substitution of hydroxyl groups on B-ring plays an important role in binding of flavones to CYP2C9. 
Table 1. Binding energy of 10 polyphenols

\begin{tabular}{lcccc}
\hline Compounds & HBD & HBA & $\begin{array}{c}\text { Mean Binding Energy } \\
(\mathrm{kcal} / \mathrm{mol})\end{array}$ & $\begin{array}{c}\text { Lowest Binding Energy } \\
(\mathrm{kcal} / \mathrm{mol})\end{array}$ \\
\hline Naringenin & 3 & 5 & -22.70 & -20.99 \\
Chrysin & 2 & 4 & -20.26 & -21.18 \\
Apigenin & 3 & 5 & -25.27 & -24.53 \\
Luteolin & 4 & 6 & -31.16 & -31.67 \\
Acacetin & 2 & 5 & -21.76 & -22.18 \\
Galangin & 3 & 5 & -13.41 & -13.59 \\
Kaempferol & 4 & 6 & -16.88 & -17.20 \\
Quercetin & 5 & 7 & -21.41 & -22.37 \\
Myricetin & 6 & 8 & -22.04 & -22.55 \\
Isorhamnetin & 4 & 7 & -13.62 & -16.70 \\
Flurbiprofen & 1 & 3 & -10.03 & -10.27 \\
\hline
\end{tabular}

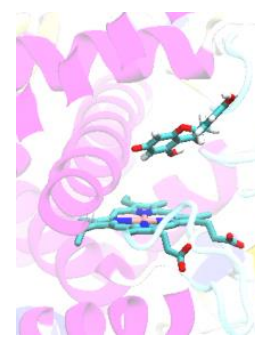

A

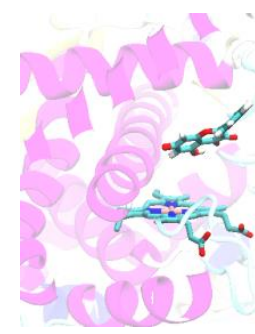

F
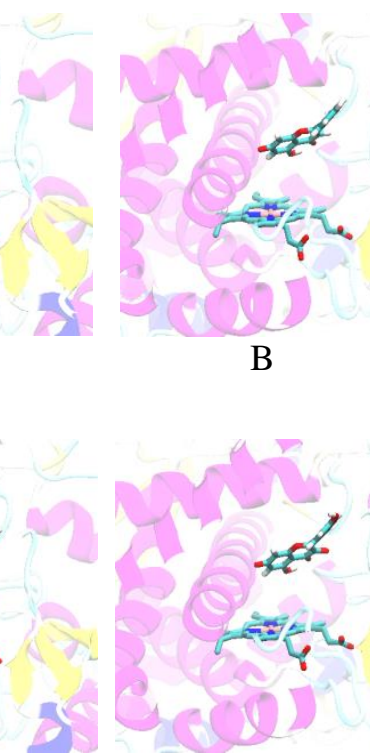

G

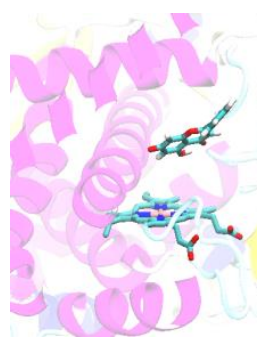

B

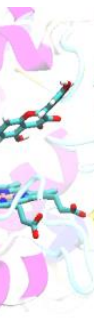

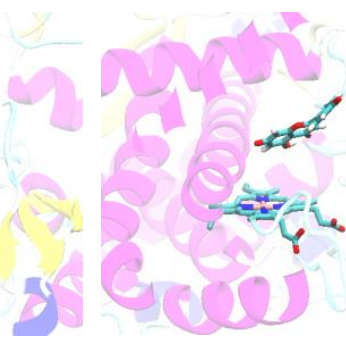

C

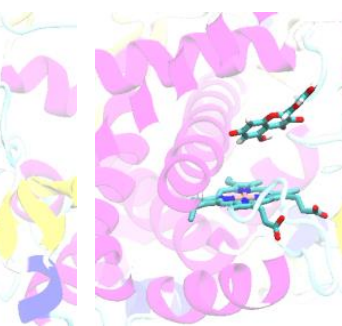

$\mathrm{H}$

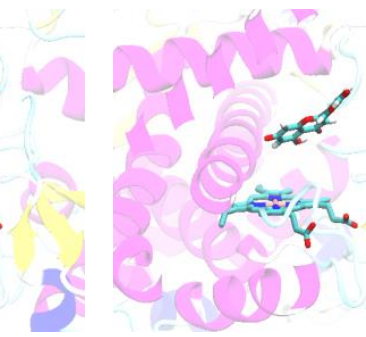

D

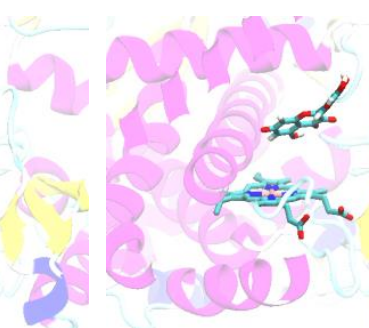

I

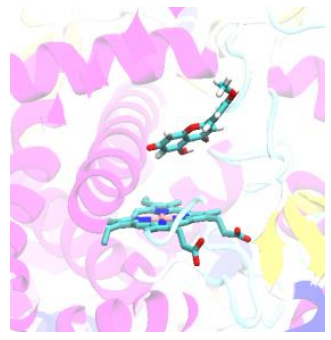

E

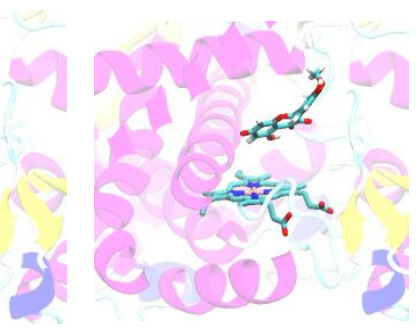

J

Figure 2. The orientation of ten polyphenols in the active site of CYP2C9; (A) naringenin, (B) chrysin, (C) apigenin, (D) luteolin, (E) acacetin, (F) galangin, (G) kaempferol, (H) quercetin, (I) myricetin, and (J) isorhamnetin.

Our docking resultshere can predict the interaction between the polyphenols and amino acid residues in the active site of CYP2C9, including the pi-pi interaction between aromatic ring $\mathrm{B}$ of polyphenols and the Phe 100 residue.

Furthermore, water molecules often play important roles for drug-enzyme interactions [12]. An water molecule (W600) is located between the haem iron and flurbiprofen at less than $3 \AA$ and potential hydrogen bonding interactions with the carbonyl of Ala29 [7].
Hence, a water molecule in active site may form hydrogen bond with the polyphenols.

\section{Conclusions}

From molecular docking study suggested that the amino acid Phe100, Pro101, Glu104, Phe114, Leu208, Gly296, Ala297, Leu366 and Phe476 residues were identified as important residues in the CYP2C9 active binding site. The binding model can be applied to predict the interaction between other polyphenols and CYP2C9. 
Therefore, further studies should be carried out using other docking programs to improve accuracy of binding models.

\section{Acknowledgement}

The docking program in this study was supported by Thailand National Electronics and Computer Technology Center (NECTEC).

\section{References}

[1] K.B. Pandey and S.I. Rizvi, Plant Polyphenols as Dietary Antioxidants in Human Health and Disease, Oxidative Medicine and Cellular Longevity, 2(5), 2009.

[2] C. Manach, et al., Polyphenols: food sources and bioavailability, The American Journal of Clinical Nutrition, 79(5), 2004, 727-747.

[3] I.C. Arts and P.C. Hollman, Polyphenols and disease risk in epidemiologic studies, The American journal of clinical nutrition, 81(1), 2005, 317S-325S.

[4] G.W.M. Chang and P.C.A. Kam, The physiological and pharmacological roles of cytochrome $\mathrm{P} 450$ isoenzymes, Anaesthesia, 54(1), 1999, 42-50.

[5] P.R.O. De Montellano, Cytochrome P450: structure, mechanism, and biochemistry, 2005: Springer.
[6] S.F. Zhou, et al., Substrates, inducers, inhibitors and structure-activity relationships of human Cytochrome P450 2C9 and implications in drug development, Curr. Med.. Chem., 16(27), 2009, 3480-675.

[7] M. R. Wester, et al., The structure of human cytochrome p450 2c9 complexed with flurbiprofen at 2.0-å resolution, Journal of Biological Chemistry, 279(34), 2004, 3563035637.

[8] G. Wu, et al., Detnhtnjmhtfailed analysis of grid-based molecular docking: A case study of CDOCKER-A CHARMm-based MD docking algorithm, Journal of Computational Chemistry, 24(13), 2003, 1549-1562.

[9] Y. Kimura, et al., Inhibitory effects of polyphenols on human cytochrome P450 3A4 and 2C9 activity, Food and Chemical Toxicology, 48(1), 2010, 429-435.ies for development of dengue virus inhibitors, Antiviral Research.

[10] D. Si, et al., Mechanism of CYP2C9 Inhibition by Flavones and Flavonols, Drug Metabolism and Disposition, 37(3), 2009, 629-634.

[11] S. Martens and A. Mithöfer, Flavones and flavone synthases, Phytochemistry, 66(20), 2005, 2399-2407.

[12] R. Wang, L. Lai, and S. Wang, Further development and validation of empirical scoring functions for structure-based binding affinity prediction, Journal of Computer-Aided Molecular Design, 16(1), 2002, 11-26. 